\title{
Research on the Adaptive Sliding Control of Permanent Magnet Linear Synchronous Motor in Neural Network
}

\author{
Hongjun Zhu \\ Zhejiang Industry Polytechnic College \\ Shaoxing, China
}

\begin{abstract}
When the permanent magnet linear synchronous motor (PMLSM) direct drive system, the system has the characteristics of nonlinear, motor parameter time-variance and vulnerable disturbance, an adaptive sliding mode controller based on neural network is designed. The controller consists of two neural networks, one is used for sliding equivalent control and the other for sliding switch control, so as to effectively guarantees the system fast tracking performance for a given signal. The controller can restrain the system parameters perturbation and external resistance changes, and weakening the sliding mode control system chattering. The simulation results show that the adaptive sliding mode controller based on neural network can improve the system robustness for parametric perturbation and external disturbance, and possess better dynamic stability and tracking performance
\end{abstract}

Keywords- Linear motor, Neural network, Sliding control, Chattering

\section{INTRODUCTION}

Permanent magnet linear synchronous motor has the advantages of large thrust, low inertia, fast response, and it is widely used in aerospace field, industrial automation, NC machine tools, robotics and special processing. But PMLSM is a multivariable and strong coupling, nonlinear, variable parameter complex object, linear motor drive directly load, load variations and external disturbances will directly influence the servo system performance. Modern control theory by using model reference adaptive control, fuzzy control and neural network control can effectively improve the performance of the motor. Neural network control has strong self-learning ability and good adaptability, can design excellent controller and enables the system has strong robustness for uncertainty disturbance during the guarantee of quick tracking performance[1].

Sliding model variable structure control appeared in the $1950 \mathrm{~s}$, is a kind of effective comprehensive method for nonlinear uncertain system. Variable structure control system has very strong robustness for the system parameter perturbation and external disturbance, and the structure is simple, response is rapid. But, the system has many uncertain factors, adopts pure sliding mode control will exist serious chattering phenomenon[2]. A method based on neural network of sliding model variable structure control method is put forwarded, using neural network realize respectively equivalent control and sliding switch control, through online adjustment, realize the sliding mode control in movement, thus effectively weaken chattering, optimize the systematic tracking performance.

\section{MATHEMATICAL MODEL}

Using the vector control method of id $=0$, in rotating $d-p$ coordinate, PMLSM state equation are as follows:

$$
\begin{gathered}
\frac{d x}{d t}=v \\
\frac{d v}{d t}=\frac{k_{f}}{m} i_{q}-\frac{B}{m} v-\frac{F_{l}}{m} \\
\frac{d i_{d}}{d t}=\left(u_{d}-R i_{d}+\omega L_{q} i_{q}\right) / L_{d} \\
\frac{d i_{q}}{d t}=\left(u_{q}-R i_{q}-\omega L_{d} i_{d}-\omega \lambda_{f}\right) / L_{d}
\end{gathered}
$$

In the formula, ud is d axial voltage, uq is q axial voltage, id is $\mathrm{d}$ axial windings current, iq is q axial windings current, $\lambda \mathrm{d}$ is $\mathrm{d}$ axial windings magnetic chain, $\lambda \mathrm{q}$ is $\mathrm{q}$ axial windings magnetic chain, $\mathrm{Ld}$ is $\mathrm{d}$ axial inductance, $\mathrm{Lq}$ is $\mathrm{q}$ axial inductance, $\mathrm{m}$ is rotor weight, $\mathrm{B}$ is viscous friction coefficient, $\mathrm{Ft}$ is load, $\mathrm{R}$ is primary winding resistance, $\lambda \mathrm{f}$ is permanent magnets magnetic chain, $\omega=\pi v / \tau, v$ is rotor linear velocity, $\tau$ is polar distance.

\section{Sliding Mode CONTROL BASED ON NeURAL NETWORK}

Conventional sliding mode control consists of two parts, the equivalent control and switch control[3], and they can show by the following formula,

$$
\begin{gathered}
u=u_{e q}+u_{s}=u_{e q}+\beta \operatorname{sgn}(s) \\
\operatorname{sgn}(s)=\left\{\begin{array}{cc}
1 & s>0 \\
-1 & s<0
\end{array}\right.
\end{gathered}
$$

In the formula, ueq is sliding mode equivalent control part, its physical meaning is switch control average, control the system determine part, us is the switch part for sliding mode control, the uncertainties by high-frequency switch control make the system state tendency, and ensure the sliding hyperplanes along the sliding surface sliding into state stable points, $\beta$ is positive and in direct proportion to general disturbance amplitude.

Equivalent control equates to system inverse dynamics. When the lack of understanding the system parameters, the equivalent to calculate gains control signal will deviating from the practice value. In order to solve this problem, adopts two neural networks to realize respectively equivalent 
control and switch control, the structure is shown in figure 1, including neural network to realize $\mathrm{NN} 1$ equivalent control, neural networks NN2 realize switch control. When model mismatch and the existence of unmodeled dynamics system sliding surface, track deviate by us this discrete system control make the system tracks trend sliding motion. Through the neural network weights updates to compensate for the sliding deviation quantity, so as to reduce the chattering.

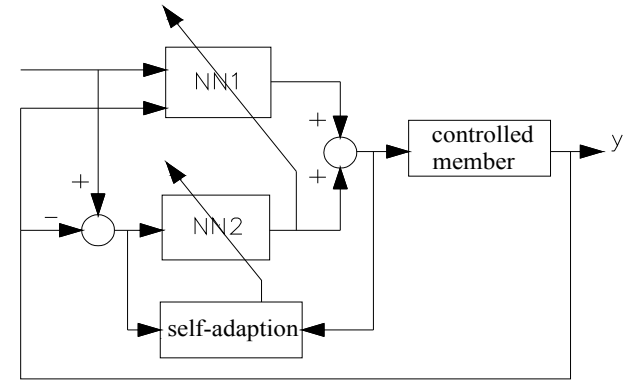

Figure 1. Structure diagram of sliding mode control system based on neural network

\section{A. Equivalent control based on neural network.}

When the system state in sliding mode control surface, equivalent control realizes the normal operation of the system, and the switch control volume is 0 . But when the system state deviation sliding surface, switch control is necessary. NN1 training objective function is the error square for target output and actual output. On the basis of formula 5 , the error can be instead of the sliding mode control switch control items[4], so

$$
E=0.5\left(u_{x}\right)^{2}
$$

NN1 choose two layers of feed forward neural network, its input and output can decide according to the formula of ueq, and the hidden and output layer adopts sigmod activation function

$$
f(x)=\frac{1}{1-e^{-x}}
$$

The general BP algorithm are easy to form local minimal and convergence slow, therefore the variable driving quantity BP learning algorithm is adopted to train the networks, the updating algorithms of weights $\mathrm{k}$ are as follows[5],

$$
\begin{gathered}
\omega(k+1)=\omega(k)+\eta \frac{\partial E}{\partial \omega}+\alpha \Delta \omega(k) \\
\left\{\begin{array}{cc}
\eta=\eta^{*}\left(1+\frac{c}{p}\right) & (\alpha=\alpha, \Delta E<0) \\
\eta=\eta_{0} & (\alpha=0, \Delta E>0)
\end{array}\right. \\
\Delta E=E(k)-E(k-1)
\end{gathered}
$$

In the formula, $\mathrm{c}$ is constant, $\mathrm{p}$ is learning cycle number.

The algorithm is to make learning rate $\eta$ and momentum $\alpha$ are variable. If the current error gradient modified direction is correct, it increases learning rate and joined the momentum, otherwise, dump momentum and reducing learning rate, so as to make the learning efficiency greatly increased.

\section{B. Switch control based on neural network.}

The sliding mode control chattering is mainly caused by control amount of high frequency switching control us. The gain of switch control us is in direct proportion to generalized disturbance amplitude, and in direct proportion to the chattering range. In PMLSM drive system, there are lots of uncertain disturbance, which makes conventional sliding mode control is difficult to do the job. Therefore, this paper using neural network on-line adjusting to compensate for control quantity is adopt to avoid generate highfrequency switch.

NN2 adopts two layer structure, a hidden and an output layer. Input electes the system state error, hidden neurons number is equal to the number of the sliding function. The output is switch control. Hidden layer choose symmetric type Sigmod function

$$
\begin{gathered}
u_{s}=\beta g(s) \\
g(s)=\frac{1-e^{-s}}{1+e^{-s}}
\end{gathered}
$$

According to formula 12, each input and hidden of NN2 is not whole connections, and connected only to a neuron of hidden layers, but the hidden layer connection to the output layer. The output layer only realizes signal superposition and no activation function.

The objective function of NN2 training is

$$
J=\frac{1}{2}\left(s^{T} s+u_{s}{ }^{T} u_{s}\right)
$$

Selecting the above formula as the objective function is the cause of the error function is $\mathrm{s}$, optimizing $\mathrm{s}$ function can make the system error is minimal. On the other hand, optimize us can eliminate chattering and realize robust control.

NN1 learning algorithm is also used to update weights, $\beta$ can update through the following formula

$$
\frac{d \beta_{i}}{d t}=-\gamma \frac{\partial J}{\partial \beta_{i}}
$$

\section{PMLSM SLIDING CONTROL BASED ON NEURAL NETWORK}

With the above sliding mode control based on neural network method to realize PMLSM drive control, system structure is shown in figure 2. NN1 output of $d$ and $q$ shaft voltage equivalent control quantity, $\mathrm{NN} 2$ output for $\mathrm{d}$ and $\mathrm{q}$ shaft voltage switch control volume. Because magnetic and speed are respectively controlling, so choose the following two sliding surfaces, 


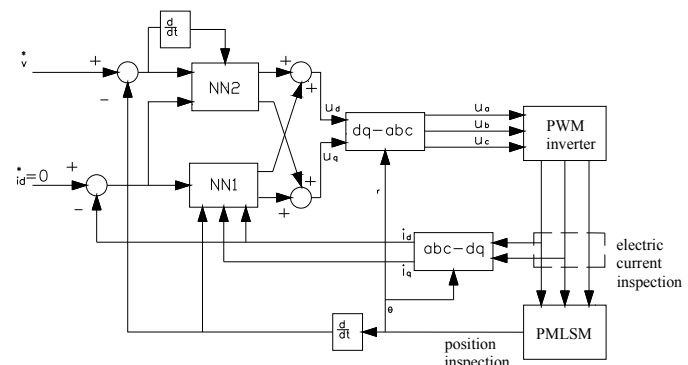

Figure 2. PMLSM sliding mode control system based on neural network

$$
\begin{aligned}
& s_{1}=i_{d}^{*}-i_{d} \\
& s_{2}=\dot{e}+\lambda e
\end{aligned}
$$

In the formula, $\mathrm{e}=\mathrm{v}^{*}-\mathrm{v}, \lambda$ is constant.

Do not break general, set $\mathrm{v}$ as the phase step given signal, the derivation of two formulas is

$$
\begin{aligned}
& \dot{s}_{1}=-\left(u_{d}-R i_{d}+\omega_{e} L_{q} i_{q}\right) / L_{d} \\
& \dot{s}_{2}=\frac{-k f}{L_{d} M}\left[u_{q}-R i_{q}-\omega L_{d} i_{d}-\omega \lambda_{f}\right]-\left[\frac{B}{M}+\lambda\right] \dot{v}
\end{aligned}
$$

Based on equivalent control method, make $s=0$, then can get $\mathrm{d}$ axis and $\mathrm{q}$ axis of voltage equivalent control component respectively

$$
\begin{gathered}
u_{d(e q)}=\frac{R}{L_{d}} i_{d}-\frac{\omega L_{q}}{L_{d}} i_{q} \\
u_{q(e q)}=\frac{M L_{q}}{k_{f}}\left(\frac{B}{M}-\lambda\right) \dot{v}+R i_{q}+\omega\left(L_{d} i_{d}+\lambda_{f}\right)
\end{gathered}
$$

Thus the $\mathrm{d}$ axis and $\mathrm{q}$ axis control voltage can be obtained

$$
\begin{aligned}
& u_{d}=u_{d(e q)}+\beta_{1} g\left(s_{1}\right) \\
& u_{q}=u_{q(e q)}+\beta_{2} g\left(s_{2}\right)
\end{aligned}
$$

$\mathrm{NN} 1$ and NN2 weights updated using mentioned methods. In order to ensure the stable operation of the system, NN1 is adopts the offline training. The sliding surface determined samples are obtained adopting the time optimal method.

\section{SimULATION RESEARCH}

In order to verify the effectiveness of the control method, matlab/simulink software is adopt to for simulation. The simulation using PMLSM parameters are respectively, $\mathrm{R}=$ $0.25 \Omega, \mathrm{Ld}=\mathrm{Lq}=2.67 \mathrm{mH}, \mathrm{kf}=89.25, \tau=16 \mathrm{~mm}, \mathrm{~m}=30 \mathrm{~kg}$, $\mathrm{B}=4 \mathrm{Ns} / \mathrm{m}, \lambda=0.1 \mathrm{~Wb}$. Select Laplace domain signal as input, add load disturbances $200 \mathrm{~N}$ at $\mathrm{t}=0.17 \mathrm{~s}$ and add weight block on rotor to make its weight into the 2 times of nominal value.

NN1 offline training neural network using Matlab toolbox completed. Initial weight of the NN2 is the random value between 0 and 1 , the system simulation sampling time is $0.002 \mathrm{~s}$.
The system simulation results are shown in figure 3 , figure (a) is the system speed outputs curve of common sliding mode control, figure (b) is the speed outputs curve of sliding mode control based on neural network.

The figure 3 (a) shows that when system is disturbed, the sliding mode control system based on neural network has good speed tracking performance, the rapid transition process, and strong robustness. And compared with figure 3 (b), the speed chattering is obvious decreases.
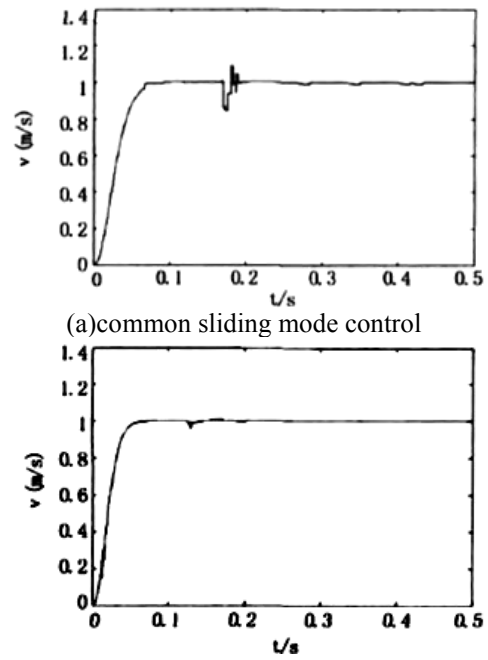

(b) sliding mode control based on neural network

Figure 3. Simulation results

\section{SUMMARIES}

Permanent magnet linear synchronous motor directly feeding system have strong nonlinear, as well as uncertainty factors such as the system parameter perturbation, load disturbance makes traditional control method is difficult to get good control performance. According to the characteristics analysis of permanent magnet linear synchronous motor, combining advantages of sliding mode variable structure control and neural network control, a neural network adaptive sliding mode controller is designed. The simulation results indicate that compared the neural network sliding mode control with conventional sliding mode control, it has better tracking performance, dynamic stability and has strong robustness of outside interference.

\section{REFERENCES}

[1] Chung S K, Lee J S. Robust speed control of brushless directdrivemotor using integral variable structure control[J], IEE Proc-Electr, 1995, 142(06), p.361-370

[2] Zou Jihao. High Performance Motion Control of Sensorless Interior Permanent Magnet Linear Synchronous Motor Control [C], Automation, Robotics and Vision Conference, 2004, (03), p.17801785

[3] Lin Faa-Jeng,Waib Rong-Jong. Robust Recurrent Fuzzy Neural Network Control for Linear Synchronous Motor Drive System[J], Neurocomputing, 2003, 50(05), p.365-370

[4] Lin Faa-Jeng,Shen Po-Hung. A Linear Synchronous Motor Drive Using Robust Fuzzy Neural Network Control[C], Fifth World 
Congress on Intelligent Control and Automation, 2004, (05), p.43864390
[5] Guo Hai-Jiao, Sagawa S. Sensorless Driving Method of Permanentmagnet Synchronous Motors Based on Neural Networks[J], IEEE Transactions on Magnetics, 2003, 39(05), p.3247-3249

Published by Atlantis Press, Paris, France.

(C) the authors 